\title{
Emergent Functions in Intranet Information Management
}

\author{
Ewa Zimmerman $^{1}$ and Björn Cronquist ${ }^{2}$ \\ ${ }^{1}$ Halmstad University, P O box 823, S-301 18 HALMSTAD, SWEDEN \\ Ewa.Zimmermandide.hh.se \\ ${ }^{2}$ Kristianstad University, SE-291 88 KRISTIANSTAD, SWEDEN \\ Bjorn.Cronquist@mna.hkr.se
}

\begin{abstract}
Organizations and municipalities implements advanced information technology, intranets, in order to enhance information management within the organization. This technology is implemented with great reliance on the technology aspect. New functions emerge as a consequence of technology, functions not planned for. This paper puts focus one such function, the key persons responsible for publishing on intranets. A descriptive study shows that these persons do exist and describes some problems they appreciate.
\end{abstract}

\section{Introduction}

Organizations and municipalities today implement computer based systems e.g. Intranets for spreading information between individuals and departments. Organizations and municipalities acknowledge intranets as an information tool that they can use for distribution of their information and to enhance inter-organizational co-operation both within departments and between governmental agencies. One basic assumption is the belief that every member and citizen use and adopt the technology.

There is lots of information on the Internet and on Intranets, some is well organized and well structured, but much is pretty chaotic which creates real problems for people who need and rely on it. Knowing how to create information that people can use and rely on is within an area that has come to be known as knowledge management. Knowledge management supports the creation, archiving, and sharing of valued information, expertise, and insight within and across communities of people and organizations with similar interests and needs (Rosenberg, 2001). Following the implementation of intranets in municipalities new information architectures have emerged without being planned for. And new functions within these architectures have emerged. When we use the term emergent we refer to tasks and positions in the expressed environment that occurs without any considerations during and after the implementation process. The need for information in organizations today is vital for their survival and the need for access to the "right" information fast is growing (Castells 1996). This paper puts focus on one obvious and significant function in line with digital information distribution; the key-persons responsible for publishing information on the intranet.. The limitations in this paper are that we focus on the key person and his/hers tasks regarding the intranet and the situation connected to the publishing process. We are only briefly discussing the intranet content e.g. what kind

The original version of this chapter was revised: The copyright line was incorrect. This has been corrected. The Erratum to this chapter is available at DOI: 10.1007/978-3-540-44836-5_33

M.A. Wimmer (Ed.): KMGov 2003, LNAI 2645, pp. 186-191, 2003.

(C) Springer-Verlag Berlin Heidelberg 2003 
of information. We are not discussing intranet functions only the obstacles the study stress regarding the publishing process. Electronic networks like electronic mail, computer conferencing and groupware are expected to enable employees to work more flexible, exchange experiences and to collaborate more effectively (Orlikowski, Yates, Okamura, Fujimoto, 1999). A group communication technology suggests that the effects of the new technology are driven by the ability to implement it into the structure of the organization/ municipalities (Weick, 1990). If this had been achieved the emergence of the key persons never had been a problem.

\section{Theory}

\subsection{Intranets}

Intranets are built around the powerful idea that web technology can be used within the organizations to facilitate communication and to provide an effective knowledge repository. Therefore, the intranet must become a valuable resource to the employees to increase the efficiency and promote knowledge sharing. At its best, the intranet forms the core of information and knowledge management efforts in the organization enabling wider strategic goals to be met, (Robertson, (2002). To make the intranet part of the daily activities must be the primary goal; to become a normal part of employee's daily lives. If a strong demand can be achieved from the users, it will create a strong incentive to support the intranet and many complaints if it is not kept up to date. It is a "critical mass" of usage that is necessary to make the intranet viable in the long-term. There is no one feature, tool or set of pages that universally makes an intranet desirable to the employees. Instead, each organization and municipalities has its own, unique set of requirements and demands. Only by involving all users in the design and evolution of the intranet will these key features be uncovered.

We describe information as a message, in the form of documents, written communication with different purposes. Information is a message addressed to everyone or someone in the organization, and the information moves around in the organization through the traditional systems (paper format) and though the new systems (electronically) e.g. intranets, (Davenport and Prusak 1998).

\subsection{Information Quality}

A framework with four IQ categories, developed through empirical studies (Huang, Lee et al. 1999), could be applied here. The first category is intrinsic $I Q$ that denotes that information has quality in its own right. Contextual IQ highlights the requirement that IQ must be considered within the context of the task at hand; that is, information must be relevant, timely, complete and appropriate in terms of amount so as to add value. Representational $I Q$ and accessibility $I Q$ emphasize the importance of the role of the systems and the systems must be accessible but secure. The systems must present information in a way that is interpretable, easy to understand, and concisely and consistently represented. These four categories should be considered in information consumer's perspective. 


\subsection{Approaches to Information or Knowledge Distribution}

Information distribution can be described in four general modes (Dixon 2000).

1. Sending everything to everyone

2. Routing the material by the collector

3. Applying central control on distribution

4. Creating a pull system where information consumers decide what they want to see Each of these systems has drawbacks. Sending everything to everyone generates an information overload and will hardly enhance the ability to get attention to and prioritize significant bits of information. Routing information might lead to placing decisions with people not qualified to make them. Applying central control generates needs of administrative support and will increase overhead by creating a central office. The fourth is, in our view, the most hazardous because it assumes that information consumers know what is available before they see the material. This can only be true for very formal and generalized artifacts of information.

Regardless of individual contexts it can be stated that in any organization, suffering from some degree of internal anonymity, the informal evaluation and transmission of information quickly creates gridlocks. Based upon thoughts and theories presented above following questions can be formulated. How do the key person, the publisher perform their task, collect and distribute information using the intranet?

\section{Research Problem and Objective}

The purpose of the research presented in this paper is to investigate the key persons and their roles in the organizational context of the intranet. The focus is on the difficulties that can occur when new functions in the municipalities are emerging. The emerging functions are new tasks concerning the collecting and distribution of the information, performed by these key persons.

Questions to be asked are to what extent the information published on the intranet is a dynamic source of knowledge creation (Nonaka and Takeushi 1995). To what extent it is valued and used. Are there other means of communicating information that are more trusted? Do the intranets function as an alibi for not having to inform but rather turning the responsibility to the information consumers to keep informed.

\subsection{Research Design and Methods}

We have examined municipalities in Sweden and performed interviews with the persons who have the responsibility to publish information on the intranets.

The research approach is descriptive and interpretative, and using a case study approach enables an understanding of the complex social phenomena that we are investigating. A case study allows an investigation to retain the holistic and meaningful characteristics of real-life cycles and organizational and managerial processes (Yin 1994).

The purpose with a survey investigation is to collect information that could be analyzed to identify patterns and to perform comparisons. The collected data should be used not only to describe but also to compare the answers. A survey can answer 
questions regarding, what, where, how and when but it is more difficult to answer questions about why. Our research is expected to answer both how and why and therefore the research strategy are two folded; the survey approach are used within a case study.

The main objective of the presented study was to obtain information from the persons responsible for the publishing process and about their opinions regarding their position in the organization. Our aim was not only to find out whether they were known by others in the organization, but also to discover how they performed there work. The survey contained questions regarding both general information about their working tasks but also the respondents personal opinion about some aspects of the publishing process were acquired. Briefly these questions refer to their background such as education, previous tasks, opinion about their current tasks and opinions towards the presumed users of the information.

The interviews where performed by telephone because the geographical distances. A survey interview could be described as a dialog between the interviewer and the respondents with the purpose to get information that the latter posses. The interviews were planned by choosing themes and questions related to the research area. The interview guide was built on four themes and underlying questions within those themes. The first theme focused on the identification of the person responsible for the intranet. The second theme focused on this person's background, education. The third theme had its focus on how the information was collected and published. Finally, the fourth theme concerned the opinion regarding the use of the intranet and its content. The interviews were performed by telephone and were written down during the interview, the respondent was allowed to express and discuss issues within the four themes relatively freely.

\section{Results}

The key persons expressed several difficulties in performing their tasks and some frustration was also expressed concerning this issues. Our study indicates that there is a lack of knowledge in municipalities concerning this important key person and their tasks. This function has emerged without being planned for and their performance has several obstacles that have to be solved. It has evolved with the technology which our results will show.

The first theme focused on the identification of responsible persons, the key persons. We started by identifying the key persons at the Swedish municipalities, we performed telephone interviews and asked to be connected to the responsible person for the intranet. Several of the respondents had no knowledge regarding which person to connect us to; this tells us that there is a lack of awareness of this function in the municipalities. The second theme focused on the key person's background and education. The result show that most of them had a technical education and they had been working with consultant activities, building web-sites, information secretary, culture worker and technical support. The key persons had a variety of background and educations; the connecting bound between them is that they have educations in other areas before they were given this position. The third theme focused on how the information was collected and published. Almost every key person expressed frustration regarding collecting information to publish. Administrative information 
such as handbooks, protocols, and work rules are always available on the intranet. The different departments are supposed to provide with information at a regular basis but this doesn't happen. Some of the municipalities have documented policies regarding the intranet and its content but most of them expressed a lack thereof. Finally, the fourth theme concerned their opinion regarding the use of the intranet and its content. Most of them expressed that they used e-mail to inform about new information; information in paper format was distributed to the managers in the different departments and the believed that most of the employees use the intranet. One of them said that she e-mailed a weekly letter to 350 middle-managers; it was printed and put on the bulletin board in the departments so everyone could read. The most expressed obstacles for achieving optimal use of the intranet was the accessibility to the computer technology. All municipalities expressed that there wasn't enough computers for the users and some of them had bad connections via modems. For example, one municipality had 9000 employees and only 1500 user names in the system. Our conclusions are that our study shows that there is a lack of knowledge in municipalities regarding implementation of intranets and the emergent functions that occurs. Several difficulties have to be over come if a successful use is going to be accomplished, for example, the accessibility to the technology must be increased.

\section{Discussion}

Designing and managing an intranet is not easy. Implementing the technical solutions is not enough. It can be viewed as a necessary but not sufficient prerequisite in supporting efficient and effective organizational information architecture. The overall purpose is to build foundation for timely and effective decisions and actions within the organization. One trivial but important aspect that often is neglected is the physical access to computer terminals during working hours.

New organizational behaviors develop as a consequence of implementing the technology. New tasks supported by employees forced to adapt to these new behaviors emerge within organizations without being planned for. One of these emergent roles is the key persons with publishing responsibilities. The importance of stressing this key person identified in our study is important. The very fact that they exists can be viewed as an indication of bad alignment between technical capabilities and organizational behavior. Old structures are just transferred to new media. The full potential and possibilities are not explored and developed.

Advanced information technology implies potential possibilities in achieving a useful and creative intranets supporting organizational communication. Acquisition and sharing of knowledge is a very important issue in modern organizations

\section{Further Research}

The mere existence of central functions for publishing on intranets and the anonymity of these key persons imply a weakness in fully taking advantage of the potential benefits of advanced information technology. The issue of information quality is an 
important aspect of information architecture. An extended study should address this issue. The usefulness of artifacts published and distributed by intranets should be studied. One hypothesis could be that published information merely functions as an alibi for making official decisions public. The responsibility to inform is shifting towards keeping informed. The publishers have done their part when putting documents on the intranet. It is up to the consumers to find it. Other functions made possible through intranet technology should also be attended. The Intranets could function as a facilitator for informal communication and communities of practice. In what way does technology influence communicative behavior? We hope that this paper will bring attention to these oft-neglected factors in implementation processes of intranets and encourage discussion about how to effectively address this issue.

\section{References}

1. Castells, M. (1996). Informationsåldern, Ekonomi, samhälle och kultur Band 1:Nätverkssamhällets framväxt, Daidalos.

2. Davenport, T. H. and L. Prusak (1998). Worknig Knowledge: How organisations Manage what they know, Harvard Business School Press.

3. Dixon, N. M. (2000). Common knowledge: how companies thrive by sharing what they know, Harvard Business School Press.

4. Huang, K., W. Lee, et al. (1999). Quality Information and Knowledge. New Jersey, Prentice Hall.

5. Orlikowski W.J., Yates, JA., (1994) Genre repertoire; The Structuring of Communicative Practices in Organizations. Administrative Science Quarterly. No 39 pp 541-574

6. Rosenberg, M., J., (2001) e-Learning - Strategies for Delivering Knowledge In the Digital Age. McGraw-Hill. USA.

7. Weick, K., (1995) Sensemaking in Organizations, Thousand Oaks, CA; Sage Publications Inc.

8. Nonaka, I. and H. Takeushi (1995). The Knowledge-Creating Company, Oxford University Press.

9. Yin, R. K. (1994). Case Study Research: Design and Methods, SAGE Publications inc. 